\title{
Deep Learning for Sentiment Analysis - Invited Talk
}

\author{
Richard Socher \\ MetaMind \\ richardesocher.org
}

Richard Socher is the CEO and founder of MetaMind, a startup that seeks to improve artificial intelligence and make it widely accessible. He obtained his $\mathrm{PhD}$ from Stanford working on deep learning with Chris Manning and Andrew $\mathrm{Ng}$ and won the best Stanford $\mathrm{CS} \mathrm{PhD}$ thesis award. $\mathrm{He}$ is interested in developing new AI models that perform well across multiple different tasks in natural language processing and computer vision.

He was awarded the Distinguished Application Paper Award at the International Conference on Machine Learning (ICML) 2011, the 2011 Yahoo! Key Scientific Challenges Award, a Microsoft Research PhD Fellowship in 2012 and a 2013 "Magic Grant" from the Brown Institute for Media Innovation and the 2014 GigaOM Structure Award. 